\title{
Raising Awareness for Value Creation Potential in Engineering Research
}

\author{
Luis M. Camarinha-Matos, João Goes, Luís Gomes, and João Martins \\ Faculdade de Ciências e Tecnologia, Universidade Nova de Lisboa, \\ 2829-518 Caparica, Portugal \\ \{cam,jg,lugo\}@uninova.pt, jf.martins@fct.unl.pt
}

\begin{abstract}
Raising awareness among engineering PhD students for the value creation potential of their research work is a crucial element in their education. With this aim, the doctoral conference DoCEIS'12 focused on technological innovation for value creation, challenging the contributors to analyze in which ways their technical and scientific contributions could contribute to creating value in society. The results of this initiative, which was reasonably successful, are briefly analyzed.
\end{abstract}

Keywords: Value creation, transferrable skills, sustainability, engineering education.

\section{Introduction}

The current markets turbulence and its associated waves of economic crises, combined with other relevant societal challenges such as the growing demographic unbalance, struggle to access to scarce resources, etc., call for new levels of social responsibility from the research community. In fact, when properly focused and accompanied by adequate exploitation strategies, technological research (both, fundamental and applied) and development can play an important role in value creation.

The notion of value, even when associated to sustainability concerns, should not be limited to an economic perspective. It is widely known that, particularly in the case of information technologies, they have a role of creating growth and competitiveness in economy, However, other values such as improving quality of life, users' satisfaction, social stability, or sustainability potential, are equally important.

Nowadays, a good deal of technological innovation is the result of the research works of engineering $\mathrm{PhD}$ students. As such, the issue of value creation potential should be an intrinsic aspect of any $\mathrm{PhD}$ program, and paramount for the dissemination of value-producing knowledge. Furthermore, in the future career of these students, as doctors, they will have a greater responsibility before the society, due to the leading role they will perform in technological development and management. As such, in addition to the technical and scientific education, it is important to help $\mathrm{PhD}$ students getting conscious of the potential transformational 
role they can play in society, raising their awareness for such issues. Further to the normal research question(s) they typically address, it is also necessary to ask how to create value, or what is the potential for value creation of their research work.

\section{The Role of DoCEIS}

The series of Doctoral Conferences on Computing, Electrical and Industrial Systems (DoCEIS), in addition to provide $\mathrm{PhD}$ students with a forum where they can present and thoroughly discuss their theses research work, also aims at contributing to help them acquire a number of skills that go beyond the purely technical and scientific focus. The acquisition of such soft or transferrable skills is, nowadays, identified as an important element in the structure of doctoral programs, as defended by the European Universities Association (EUA 2007). This aspect is also recognized by OECD, which recently promoted a workshop on Transferable Skills Training for Researchers (OECD 2011). As stated by Ester Basri, senior policy analyst in the OECD science and policy division, "Transferable skills help researchers pursue varied careers and contribute to better research outputs, and can ultimately enhance research and innovation performance" ${ }^{1}$.

With this goal in mind, DoCEIS has been focusing on general themes with a wide societal importance, creating the opportunity to discuss the contribution of each thesis work to the challenges involved in the proposed themes.

This edition is focused on "Technological Innovation for Value Creation". As such, all contributed papers were specifically requested to include a section discussing the potential contribution of the presented research results to value creation. The inclusion of such section provided a unifying element among works that span across a wide spectrum of technical and scientific topics within Computing, Electrical and Industrial Systems areas, also facilitating creation of synergies among different disciplines and multidisciplinary areas of research.

Since most students are not used to think in these terms, the normal tendency would be to only focus on the technical specificities of their work. It was thus necessary to consistently ask for this additional contribution during all main phases of the conference preparation:

- Announcement of the call for papers

- Paper evaluation and selection

- Preparation of camera ready version of papers.

\section{Summary of Contributions}

As a result of this effort, and in spite of the heterogeneity of the contributions and levels of objectivity, various interesting analysis could be produced. In a few cases the goal of this exercise was not fully understood, and some students focused on

${ }^{1}$ University World News, issue 185, 21 Aug 2011. 
highlighting the technical innovations with only brief references to general systems' improvement. But in most cases a useful discussion of the potential for value creation associated to the technical / scientific results is provided.

The most relevant elements of the provided discussions include:

- Systems' performance improvement.

- Achieving more cost effective solutions.

- Systems optimization.

- Improvement of systems autonomy, flexibility and reliability.

- Facilitation of collaboration.

- Reduction of development costs / time.

- Improvement of quality and clients' satisfaction.

- Improvement of independent living conditions for people with special needs.

- Improvement of learning / training support.

- Reduction of power consumption.

- Improvement of organization models, supporting business opportunities.

- Reduction of risks.

- Contribution to sustainability.

- Improvement of human-machine interaction.

- Facilitation of decision-making.

- Improvement of maintenance strategies.

The diagram presented in Fig. 1 summarizes the relative distribution of the main contributions.

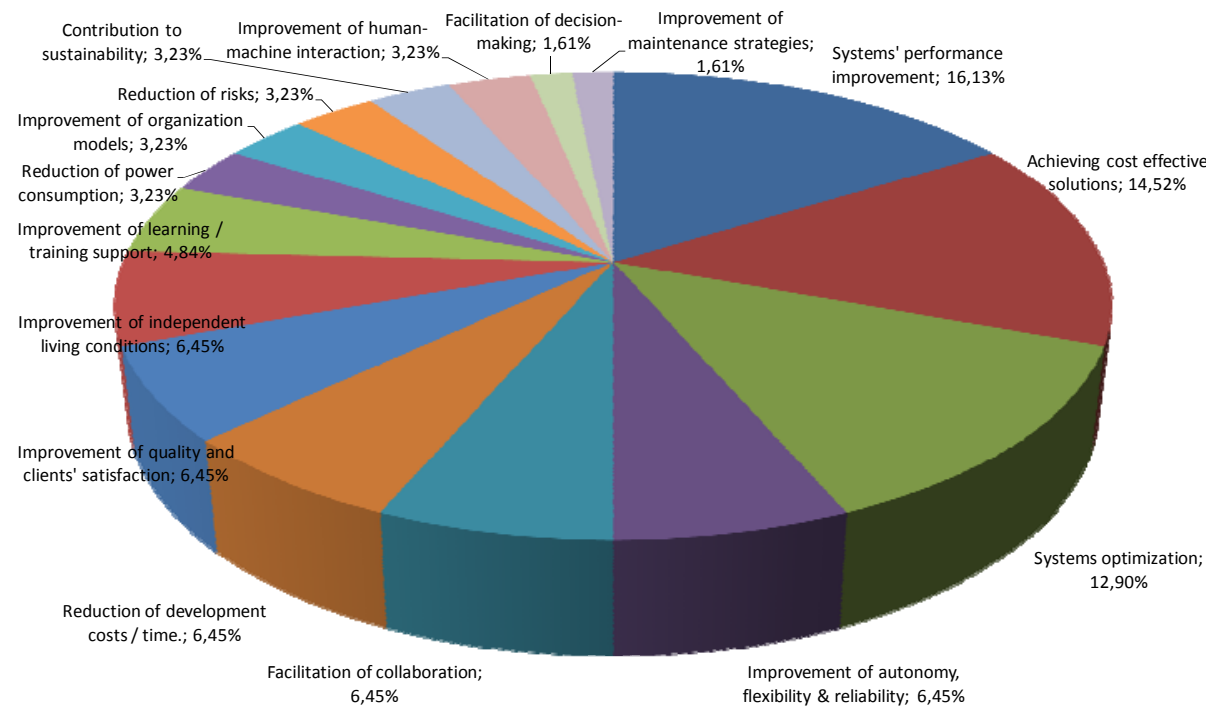

Fig. 1. Distribution of elements for value creation as proposed by $\mathrm{PhD}$ students in the different DoCEIS papers 


\section{Concluding Remarks}

A brief analysis of the discussions included in the contributed papers lets us conclude that, in most cases, the goal of challenging the $\mathrm{PhD}$ students to consider the potential for value creation associated to their research work was reasonably achieved. In this way, DoCEIS has given a modest, but important contribution to build a sounder awareness for societal challenges and the leading role that future $\mathrm{PhDs}$ in engineering can play. A paradigm shift based on a value creation approach is certainly very efficient, in the sense that most of the research activities carried out by a majority of $\mathrm{PhD}$ students must be able to be translated, later on, into valuable knowledge or/and into value creation (e.g. incorporated in an industrial product or as a service).

Similarly to other editions of DoCEIS, the papers presentation and discussion at the conference are also expected to address this important aspect.

One of the recommendations of the European Universities Association states: "An important element of transferable skills development is bringing together doctoral candidates from different disciplines and different levels (1-3 year) to encourage interdisciplinary dialogue and foster creative thinking and innovation" (EUA 2007). DoCEIS, as also reflected in the wide scope and diverse levels of progress of the accepted papers, is designed with this objective in mind. The proposition of a transversal topic in every edition, like the topic of value creation, combined with a number of other activities oriented to building transferrable skills, has proven successful and highly appreciated by participants.

\section{References}

1. EUA, Doctoral programmes in Europe's universities: Achievements and challenges. Report prepared for the European universities and ministers of higher education (2007), ISBN: 9789078997047 ,

http: / /www. eua.be/fileadmin/user_upload/files/Publications / D octoral_Programmes_in_Europe_s_Universities.pdf

2. OECD, OECD RIHR Workshop on Transferable Skills Training for Researchers: Supporting career development and research (November 28, 2011),

http: / /www. oecd.org/dataoecd/59/14/48880398.pdf 